\title{
Materials and Complexity: emergence of structural complexity in sphere packings
}

\author{
T. Aste ${ }^{1}$ and T. Di Matteo \\ Department of Applied Mathematics, Research School of Physical Sciences, The Australian \\ National University, 0200 Canberra, ACT, Australia
}

\begin{abstract}
The contemporary science of materials and condensed-matter physics is changing in response to a new awareness of the relevance of concepts associated with complexity. Scientists who design and study new materials are confronted by an ever-increasing degree of complexity, both in the materials themselves and in their synthesis. Typically, modern advanced materials are partially non-crystalline, often multicomponent, and form out of equilibrium. Further, they have functional and structural properties that are active over several length-scales. This emerging structural and functional complexity is intrinsic and necessary to many aspects of modern materials; features common also to several other complex systems. In this paper we briefly review the emerging structural complexity in a special model system: sphere packings.
\end{abstract}

Keywords: Complex systems, Granular Materials, Networks.

\section{INTRODUCTION}

A common paradigm in science tends to assume that things are naturally inclined to pursuit order and perfection. When the atomic structure of materials is concerned, such a paradigmatic order is achieved by the endless periodic repetition of local motifs in perfect crystals. Such an assumption has a quite solid background. Indeed, there are several good reasons to choose order as a constitutive paradigm. Order is convenient: the complete information about a crystalline structure can be encoded in a very compact way by retaining only the information about a single fundamental cell plus some translational symmetry operations. Order is efficient: often the most efficient structures have high degrees of symmetry and result in crystalline arrangements. But, on the contrary, disorder is everywhere: in nature order is more the exception than the rule. This is in particular the case when complex structures are concerned as for instance in several nanomaterials or in other systems

\footnotetext{
${ }^{1}$ Corresponding author: Tomaso Aste, e-mail: tomaso.aste@anu.edu.au
} 


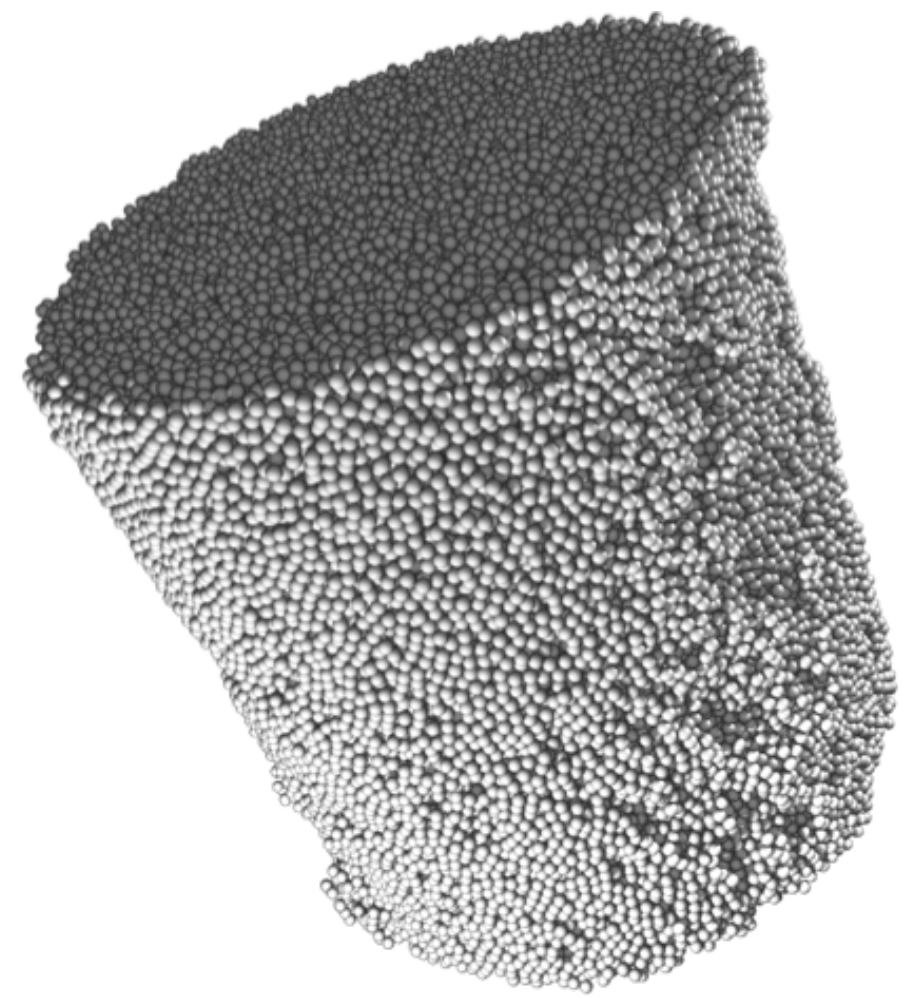

Figure 1: About 150000 equally sized spheres packed in a cylindrical container.

such as polymers, biological systems and glasses. The actual driving mechanisms that lead to such disordered structures are very diverse and range from fast out-of-equilibrium cooling (metal glasses) to evolution (biological systems). One should remark that in small systems, the competition between the contribution from the surface respect to the contribution from the bulk becomes extremely important and there exists a critical size below which an organized disorder is more effective than order, making in this way non crystalline structures favorite at nano-scales.

It must be first clarified that disorder is not necessarily randomness: we often encounter systems that are highly organized both at local and global scale but nevertheless do not reveal any periodic (or quasi-periodic) pattern. The study of such organized but disordered structures is very challenging and the available investigation tools appear to be inadequate to capture the essential features of these structures. Indeed, a complete description of the structure of a disordered system requires the information about the exact position, 
orientation, shape and connectivity of each element. However, part of such information is redundant or irrelevant and several degenerate states with different microscopic realizations can share the same macroscopic properties. To determine which are the accessible configurations at the local level, and to understand which are the possible combinations which generate the global packing is of fundamental importance. In particular, finding measures for local and global, organization is the essential starting point towards the understanding of the basic mechanisms which form these structures. It is also an essential step in the development of technologies which enable us to control and tune the structure of amorphous materials.

In this paper we focus on disordered sphere packings as an archetypal example. We review and discuss the most suitable paradigms and tools to investigate and classify the structure of such systems. We examine the 'typical' local configurations, their relative occurrences, their correlations, their organization and the resulting overall hierarchical structure.

Our study is based on the analysis of the largest empirical dataset on disordered structures presently available in the literature. Such a dataset records the positions of more than 385000 sphere centers in 6 different packings of equally sized spheres. It should be noted that, although these studies are specific to the case of equal spheres, the methodology that we use is based on the analysis of the properties and constraints resulting from the inescapable rules dictated by geometry and packings. Therefore the results are rather universal.

\begin{tabular}{lllllllll}
\hline & density $(\boldsymbol{\rho})$ & $\mathbf{N}$ & $\boldsymbol{N}_{\boldsymbol{G}}$ & $\boldsymbol{n}_{\boldsymbol{c}}$ & $\boldsymbol{n}_{\boldsymbol{t}}(\mathbf{1})$ & $\boldsymbol{n}_{\boldsymbol{t}}(\mathbf{1 . 0 2})$ & $\boldsymbol{n}_{\boldsymbol{t}}(\mathbf{1 . 0 5})$ & $\boldsymbol{n}_{\boldsymbol{t}}(\mathbf{1 . 1})$ \\
\hline $\mathbf{A}$ & $0.586 \pm 0.005$ & 102897 & 54719 & $\mathbf{5 . 8 1} \pm 0.3$ & 3.0 & 5.5 & 6.7 & 7.5 \\
$\mathbf{B}$ & $0.596 \pm 0.006$ & 34016 & 15013 & $\mathbf{5 . 9 1} \pm 0.2$ & 2.9 & 5.9 & 6.8 & 7.7 \\
$\mathbf{C}$ & $0.619 \pm 0.005$ & 142919 & 91984 & $\mathbf{6 . 7 7} \pm 0.2$ & 3.5 & 6.4 & 7.5 & 8.4 \\
$\mathbf{D}$ & $0.626 \pm 0.008$ & 35511 & 15725 & $\mathbf{6 . 7 8} \pm 0.2$ & 3.3 & 6.0 & 7.5 & 8.4 \\
$\mathbf{E}$ & $0.630 \pm 0.01$ & 35881 & 15852 & $\mathbf{6 . 9 5} \pm 0.2$ & 3.4 & 6.3 & 7.6 & 8.6 \\
$\mathbf{F}$ & $0.640 \pm 0.005$ & 36461 & 16247 & $\mathbf{6 . 9 7} \pm 0.2$ & 3.3 & 6.9 & 7.9 & 8.9 \\
\hline
\end{tabular}

Table 1: Sample density and the interval of variation $( \pm)$ within each sample; number of spheres in the sample $(N)$; number of spheres in the central region $\left(N_{G}\right)$; estimated average number of neighbors in contact $\left(n_{c}\right)$, average number of neighbors at given radial distance $\left(n_{t}(r)\right.$ with $r=1,1.02,1.05,1.1$ diameters $)$.

\section{EQUAL SPHERES PACKINGS}

A static stack of particles in a container can minimize its potential energy (under gravity) by maximizing the packing fraction. Such a pursuit of maximum compaction is common to 
several other physical systems. Notably, at atomic level this is a feature associated with metallic bonding. From a purely geometrical perspective, it is known that the largest attainable packing fraction in a system of equal spheres is $\rho=\pi / \sqrt{18} \sim 0.74$ (Conway \& Sloane 1997, Aste \& Weaire 2000) which corresponds to a disposition of the spheres in an ordered stack of parallel hexagonal layers (forming the so-called Barlow packings). On the other hand, when equal spheres are poured in a container they spontaneously arrange in a disorderly fashion occupying a fraction of the total volume (called packing fraction or packing density $\rho$ ) between 0.55 and 0.64 . As mentioned above, here we are discussing a paradigmatic example of a system where the most efficient global structure is ordered but, on the other hand, it spontaneously forms disordered arrangements. It is very interesting to note that such a disordered state is rather robust and resilient: operations such as gentle shaking or compression might change the packing fraction (within a range 0.61-0.64) but no spontaneous generation of configurations with packing fraction above $\sim 0.64$ has ever been reported. Such a packing fraction appears therefore to be an (empirical) upper limit and it is often refereed in the literature as the 'random close packing' limit. Lager densities can only be obtained by externally inducing (partial) crystallization.

Until now the empirical investigation of the geometrical structure of these systems has been limited by the very little availability of accurate experimental or numerical data. Indeed, after the seminal works of Bernal, Mason and Scott (Bernal \& Mason 1960, Scott 1962, Mason 1968), only very recently the use of tomography has allowed to 'see' three dimensional structures and explore their geometry from the sphere level up to the whole packing.

\subsection{Six samples}

All the structures discussed in this paper refer to a database constructed from the empirical analysis, by means of X-ray Computed Tomography, of several large samples of disorderly packed monosized spheres. The experimental technique and some of the empirical results were presented in a preliminary form in (Aste, Saadatfar, Sakellariou \& Senden 2004) and in a more complete form in (Aste, Saadatfar \& Senden 2005). These studies are the largest and the most accurate empirical analysis of disordered packings ever performed. The entire database collects the coordinates (with precision better than $0.1 \%$ of their diameters) of more than 385000 spheres from 6 samples of monosized acrylic spheres prepared in a cylindrical container $^{2}$. In this paper we refer to these samples as sample A, B, C, D, E and F. An image of sample $\mathrm{C}$ is reported in Fig.1. The geometrical investigation of the packing structure was performed over a central region $(\boldsymbol{G})$ at 4 sphere-diameters away from the sample boundaries. (Note that spheres outside $\boldsymbol{G}$ are considered when computing the neighboring environment of spheres in $\boldsymbol{G}$ ). The total number of spheres in each sample, the respective numbers in $\boldsymbol{G}$ and

\footnotetext{
${ }^{2}$ Additional material is available at: http: //wwwrsphysse.anu.edu.au/granularmatter/
} 
the samples densities are reported in Table 1.

\section{SEARCHING FOR STRUCTURE: THE RADIAL DISTRIBUTION FUNCTION}

The radial distribution function is the probability distribution to find the center of a particle in a given position at a radial distance $r$ from the center of a reference sphere. This measurement is widely used to characterize packing structures and contains information about long range interparticle correlations and their organization (Scott 1962, Mason 1968, Bideau \& Hansen 1993)

Figure 2 shows the normalized radial distribution functions $\tilde{g}(r)$ for the 6 samples AF (Aste et al. 2004, Aste, Saadatfar \& Senden 2005). In this figure it is clearly visible a large peak at $r / d=1$ which is due to the contribution from the spheres in contact. The presence of other peaks after this first peak at $r / d=1$ is a clear indication that the system is organized: a structure with distinct local patterns and characteristic lengths is present. For instance, it is easy to verify that a radial distance $r / d \sim \sqrt{3}$ is consistent with configurations made by placing the centers of 4 touching spheres on the vertices of two in-plane equilateral triangles which share an edge. Whereas, the peak at $r / d \sim 2$ is due to three or more spheres which are lying along a (rather) straight line.

The detail of the secondary peaks (Fig.2b) shows that both the peaks at $r / d=\sqrt{3}$ and $r / d \approx 2$ increase in height with the packing density (although with different rates (see Fig.2c)). The presence of secondary peaks is a clear indication of structural organization and the fact that these peaks sharpen with the packing fraction certainly indicates an increasing organization. On the other hand, no signs of crystallization are detected. This is discussed in the next section. 

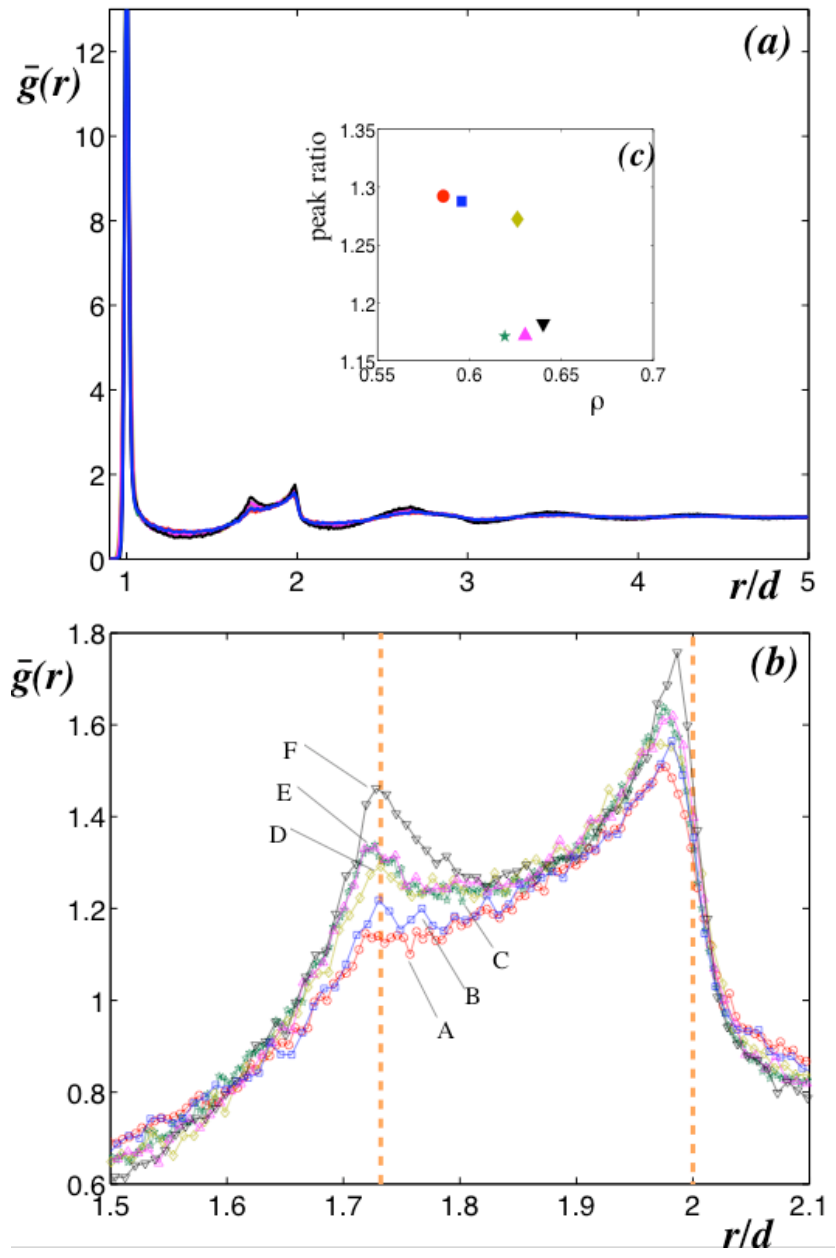

Figure 2: (a) Normalized radial distribution function. (b) The detail of the two peaks respectively at $r / d=\sqrt{2}$ and $\mathrm{r} / \mathrm{d}=2$ (vertical lines). (c) Ratio between the value of the peak at $\mathrm{r} / \mathrm{d}=2$ and the peak at $r / d=\sqrt{3}$ v.s. packing density. 


\begin{tabular}{llllllll}
\hline & $\langle f\rangle$ & $\boldsymbol{f 4}_{\mathbf{4}}(\boldsymbol{\%})$ & $\boldsymbol{f 5}_{\mathbf{5}}(\boldsymbol{\%})$ & $\boldsymbol{f}_{\mathbf{6}}(\boldsymbol{\%})$ & $\boldsymbol{p}_{\mathbf{4}}(\boldsymbol{\%})$ & $\boldsymbol{p}_{\mathbf{5}}(\boldsymbol{\%})$ & $\mathbf{5 5 5}(\boldsymbol{\%})$ \\
\hline $\mathbf{A}$ & 14.6 & 20.3 & 33.8 & 26.8 & 17.5 & 2.59 & 0.01 \\
B & 14.6 & 20.0 & 34.0 & 26.8 & 18.6 & 2.66 & 0.01 \\
C & 14.4 & 19.4 & 37.3 & 27.8 & 26.4 & 6.00 & 0.06 \\
D & 14.4 & 19.5 & 36.4 & 28.1 & 24.4 & 5.48 & 0.06 \\
E & 14.4 & 19.4 & 37.1 & 28.2 & 26.7 & 6.11 & 0.06 \\
F & 14.3 & 18.8 & 39.6 & 28.6 & 30.6 & 8.29 & 0.09 \\
\hline
\end{tabular}

Table 2: Average number of Voronoï faces $\langle f\rangle$. Fractions of Voronoï faces with 4, 5 and 6 edges $\left(f_{4}, f_{5}\right.$ and $\left.f_{6}\right)$. Fraction of configurations with 4 and 5 common neighbors ( $p_{4}$ and $\left.p_{5}\right)$. Fraction of configurations with 5 bonded common neighbors disposed in a pentagonal ring (555). This configuration can be viewed as a 'piece' of icosahedron. (The threshold distance is $1.1 d$.)

\section{UNDERSTANDING THE STRUCTURE: LOCAL GEOMETRICAL ORGANIZATION}

The radial distribution function clearly shows that despite the fact that these systems are amorphous and disordered, they have a characteristic structural organization which reveals itself with regularities at local level. However, the study of the local rotational symmetry does not disclose any known special order (Aste 2005, Aste, Saadatfar, \& Senden 2005). In this section we focus on techniques to identify local correlations which involve more than two neighboring spheres.

\subsection{Voronoï shapes}

Let us first consider the analysis of the shapes of Voronoï cells constructed around the centre of each sphere. The Voronoï cell is a polyhedron the interior of which consists of all points closer to the center of the given sphere than to any other sphere center in the sample. It coincides and generalizes the Wigner-Seitz cell construction for crystalline systems. This is a powerful tool to investigate the local arrangement of packed objects in space and it can be used to classify their local organization. The advantage of such a method is that there are no arbitrary choices for the neighboring criteria. On the other hand, this is also its weakness, 


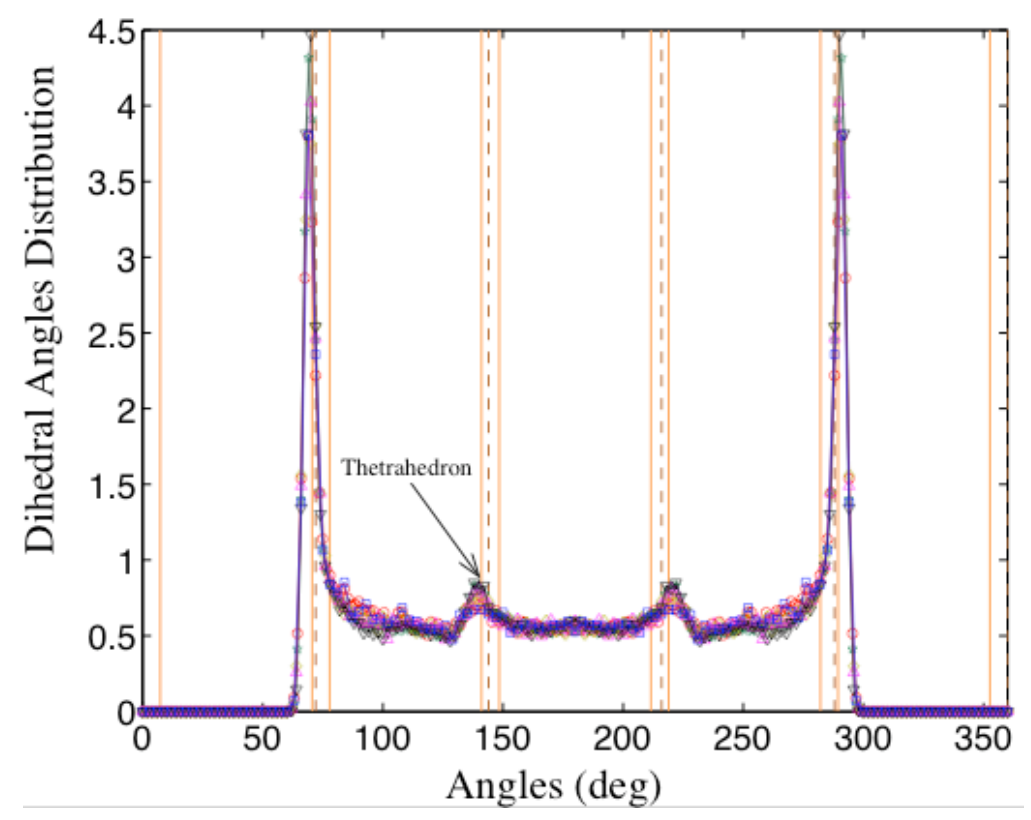

Figure 3: Dihedral angle distribution (x-axis: angular degrees; $y$-axis: renormalized frequencies). The vertical lines indicate the angles $\theta=\mathrm{n} \arccos (1 / 3)$ (and 360- $\theta$ ) with $\mathrm{n}=1,2,3,4,5$ (tetrahedral packings). The dashed lines are at the angles $\theta=n \frac{360}{5}$ $(n=1,2,3,4)$, which will correspond to icosahedral configurations.

because neighbors which are rather far from the central sphere (typically, up to distances $r / d \sim 1.4)$ contribute to the shape of the cell.

In (Aste et al. 2004, Aste, Saadatfar \& Senden 2005) we observe an average number of Voronoï faces which decreases with the density (see Table 2) from $\langle f\rangle=14.6$ at $\rho=0.586$ (sample A) to $\langle f\rangle=14.3$ at $\rho=0.64$ (sample F). Whereas the fractions of faces with 4,5 and 6 edged (respectively $f_{4}, f_{5}$ and $f_{6}$ ) follow mixed trends (see Table 2 ). These data give a clear indication that the internal structural organization of these systems is changing with the overall packing density. However, these measures are still too little sensitive to clearly define a precise link between the local properties of the packing structure and the overall packing density.

\subsection{Common Neighbors}

An alternative interesting approach called 'common-neighbor analysis of structure' was introduced by Clarke and Jónsson (1993). This analysis is based on the study of local 'bonds' 
between particles. In particular, two spheres are 'bonded' if they stay within a given threshold radial distance. The study consists in analyzing the structure around couples of bonded particles identifying common neighbors which are bonded to both the particles. In general, two touching equal spheres can have a number between 0 and 5 of common neighbors which are also in touch with both spheres. This number is rather sensitive to the local correlations in the packing. Indeed, in (Aste 2005) it is reported that the numbers of configurations with 4 or 5 common neighbors increase sensibly during compaction (see Table 2). In particular, when the threshold distance is fixed at $1.1 d$, the fraction of edges with 4 common neighbors $\left(p_{4}\right)$ varies between 17 and $31 \%$; whereas the configurations with 5 common neighbors $\left(p_{5}\right)$ grow from less than $3 \%$ to above $8 \%$ (A to F). This is in agreement with what observed in computer simulations and it suggests that these quantities could be profitably used as 'order' parameters to characterize the packing structure. Indeed, they are much more sensitive than the equivalent quantities obtained from the study of the shape of the Voronoï cell or the $Q_{4}$ and $Q_{6}$ measurements (Aste 2005).

\subsection{Searching for close local packings}

We have previously discussed that the closest local arrangement of equal spheres in three dimensions is achieved by 12 spheres in touch with a central sphere and placed on the vertices of a regular Icosahedron. We have also shown in another paper (Aste 2005) that in the samples A-F there are no traces of icosahedral rotational symmetry. However, this does not exclude the possibility that close packed configurations made by 'pieces' of icosahedron might be present in these structures. Signatures of icosahedral order can be singled out by analyzing in which way common neighbors arrange themselves around the two touching spheres. In particular a configuration of 5 bonded spheres arranged in a pentagonal ring around a couple of bonded spheres is a local signature of a (partial) icosahedral order. Such a configuration is indeed a 'piece' of icosahedron and therefore its occurrence within the samples is a good measure to understand whether icosahedral configurations might have played any significant role in the dynamical formation and in the static organization of such structures.

We observe (Aste, Saadatfar \& Senden 2005) that such configurations are present only in very small fractions (from 0.01 to $0.1 \%$, see Table 2), indicating therefore that this kind of closed packed local arrangements have very little statistical significance and no relevant physical importance. This observation is in agreement with the results from the $\left(Q_{4}, Q_{6}\right)$ analysis (Aste 2005). It appears therefore that, differently from the two dimensional case, in three dimensions closed packing configurations are not present - even at local level. A further demonstration of this comes from the study of the local volume fractions. Over a total number of more than 209000 local Voronoï configurations, only 14 have volume fractions above 0.74 (the $f c c$ has $0.7405 \ldots$ and the icosahedral arrangement has $0.75 \ldots$ ). A similar evidence can be obtained by looking at the local density associated with the Delaunay 
tetrahedral decomposition. Such decomposition is constructed by choosing the vertices of each tetrahedron on the centers of four neighboring spheres under the condition that no other spheres in the pack have centers within the circumsphere of the Delaunay simplex. We find that across the samples A-F only $2 \%$ of Delaunay configurations are closed tetrahedra. This is a further evidence that closed packed configurations play no relevant role in the structure of these systems. This therefore also implies that geometrical frustration, resulting from local dense packing, incompatible with global translational symmetry, cannot have any relevant role in the mechanism of formation of these disordered systems.

\subsection{Dihedral angles distribution}

A further step toward the understanding of which kind of local configurations are present in these systems can be undertaken by analyzing the distribution of dihedral angles between common neighbors around a given couple of bonded spheres. Previously we have calculated the number of common neighbors and shown that the most frequent configurations are the arrangements with 4 and 5 common neighbors. Let us here study how these spheres are distributed inside this common ring. To this end, we first construct the triangle between two bonded spheres and one common neighbor and then we measure the dihedral angles between such a triangle and all the other triangles formed with the other common neighbors. The resulting distribution of angles is shown in Fig.3. This figure shows a distribution which is symmetric in $\theta$ and $360-\theta$. In such a distribution, the largest peak is at $\theta=\arccos (1 / 3)=70.5 \ldots$ (and $360-\arccos (1 / 3)=289.4 \ldots$ ) which is the dihedral angle of a regular tetrahedraon. Another (smaller) peak is visible at $\theta=2 \arccos (1 / 3)=141.0 \ldots$ (and 218.9..) which corresponds to a configuration with two touching tetrahera. This clearly indicates that the common neighbors tend to gather together in the circular ring forming tetrahedral packings with angles $\theta=n$ $\arccos (1 / 3)$. Indeed, the case with an uniform angular repartition of common neighbors will lead to a rather different structure of the angular distribution with peaks at $n \frac{\pi}{3}, n \frac{\pi}{4}$ and $n \frac{\pi}{5}$, with $n=1,2,3,4 \ldots$. These peaks are less visible than the 'tetrahedral' ones in the empirical distributions in Fig.3. It is interesting to note that, similarly to the case for the radial distribution function, the relative heights of the peaks are sensitive to the sample density. But in this case the variations are less evident. 

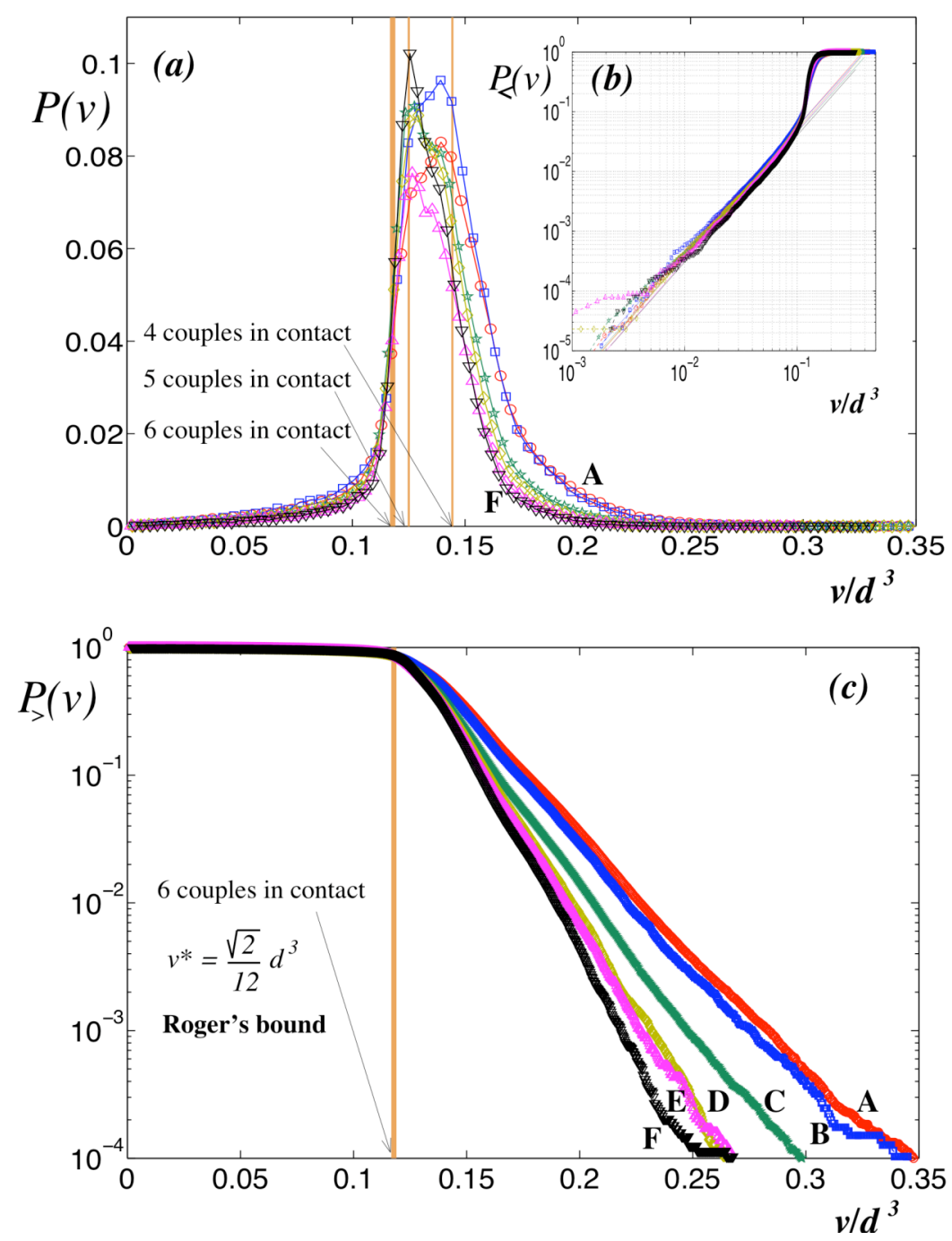

Figure 4: (a) Normalized frequencies of the distribution of Delaunay volumes in $\boldsymbol{G}$. The vertical lines indicate the maximum volumes attainable by tetrahedra with 4,5 , or 6 couples of spheres in contact.

(b) Log-log plots of the cumulative distributions $P_{<}(v)$ (the probability of finding a volume smaller than $v$ ).

(c) Log-linear plots of the inverse normalized cumulantive distribution $P_{>}(v)$ (the probability of finding a volume larger than $v$ ). 


\section{A PACKING OF TETRAHEDRA}

In the previous section, we discussed that these amorphous structures can be conveniently viewed as the result of a packing of tetrahedra. A natural way to study such a system of tetrahedra is the Delaunay decomposition where space is divided into minimal tetrahedra (with vertices on the centers of neighboring spheres and chosen in such a way that no other spheres in the pack have centers within the circumsphere of each Delaunay tetrahedron). In this section we discuss how these Delaunay tetrahedra partition space and in particular we focus on the distribution of the Delaunay's volumes. Indeed, in a given packing some local configurations are closer and others are looser and the whole packing is made by gluing together these local configurations in a disordered way which is compatible with mechanical stability, geometrical constraints and global density. The study of how space is shared among the packed spheres is essential for understanding how efficiently the spheres are arranged locally.

It has been observed (Aste 2005) that the volumes of the Delaunay tetrahedra follow distributions which, at large volumes, are well described by the exponential behavior: $P(v) \propto \exp \left(-\beta v / d^{3}\right)$, where the coefficients $\beta$ grow with the density ranging between $\beta \sim 44$ at $\rho=0.586$ (sample A) to $\beta \sim 73$ at $\rho=0.64$ (sample F) (see Fig.4).

In order to understand the origin of such exponential behavior, let us consider the whole system as an ensemble of local independent cells that freely exchange volume among each other under the constraint that the sum over all the volumes must be a constant which characterizes the state of the system. If we assume that: 1) cells are undistinguishable (the shapes are ignored); 2) there are no correlations among cells; 3) the geometrical constraints on the packing (stability, kissing number, etc.) have no relevance; then the probability to find a cell with a volume $v$ must be given by: $P(v) \propto \exp \left(-\beta v / d^{3}\right)$, with $\beta=d^{3} /\langle v\rangle$ (Aste 2005). However, this simple argument predicts coefficients which are not satisfactory: we obtain $\beta \approx 36 \rho / \pi$ which gives values for the coefficients which are one order of magnitude lower than the empirical coefficients. On the other hand, it is also evident from Fig.4 that these distributions are not pure exponentials. Indeed, they have an exponential tail at large volumes but the exponential behavior ceases at smaller volumes.

Looking in detail at Fig. 4 we observe that the exponential trend starts at values of $v / d^{3}$ around 0.14 . Such a critical volume is very meaningful. Indeed, a tetrahedron where only 4 couples of sphheres are in touch has a maximum volume $v / d^{3}=0.144$. Conversly, with when all the 6 couples spheres are in touch the volume is $v / d^{3}=0.118$; whereas, when 5 couples are in touch a tetrahedron can reach a maximum volume of $v / d^{3}=1 / 8=0.125$. The fact that the exponential ceases in this regiion of volumes indicates that the Delaunay tetrahedra can arrange freely in space only up to the limit when spheres get in contact and geometrical constraints begin to be relevant. We can therefore view these systems as comprised of two phases: 1) a 'solid' phase made by compact tetrahedra $\left(v / d^{3} \leq v^{*} \sim 0.118\right)$ which are 
geometrically constrained and are responsible for the mechanical stability; 2) a 'fluid' phase made by loose tetrahedra $(v / d>0.144)$ which are geometrically unconstrained and take volumes accordingly with the distribution which maximizes entropy. We verified that, by assuming that the exponential behavior is followed only above $v / d^{3}=1 / 8$, one can calculates analytically the coefficients at the exponent:

$$
\beta^{-1}=\frac{\langle v\rangle}{d^{3}}-v^{*} \approx \frac{\pi}{36} \rho^{-1}-0.118
$$

which returns values very close to the ones measured empirically.

\section{CONCLUSIONS}

In this paper we discussed the structure of a very simple system: the packing of equal spheres; showing that complex structural organization can emerge also from such simple aggregates. Our efforts have been devoted to discuss and uncover several specific features that are relevant for the understanding of the structure of amorphous systems. The most important concepts and the principal findings can be schematically summarized in the following points: 1) disordered is not randomness, characteristic lengths and similar local motifs are repeated (non-periodically) all over the systems; 2) amorphous systems are organized and such organization can be detected and quantified with several techniques; 3) such a structural organization extends hierarchically beyond first neighbours; 4) there are no signatures of (poly)-crystalline arrangements - even at local level.

The analyses and discussions presented in this paper concern packings of monosized spheres. Such packings have been considered as the archetypal form for amorphous arrangements of particles in space. On the other hand, different physical systems have distinct properties that are consequences of different interactions between particles and/or are the outcomes of different dynamics. However, in this paper we focused on some structural properties which are the results of geometrical and topological constraints only. Therefore, the laws and properties that we have uncovered are mostly determined by the rules governing the packing of objects in space. In the quest for universality, such laws and rules should be valid and applicable to a very broad range of systems.

\section{Acknowledgements}

Many thanks to T.J. Senden, M. Saadatfar, A. Sakellariou for several discussions and the tomographic data. We thank A. Limaye for the packing images. This work was partially supported by the ARC discovery project DP0450292 and Australian Partnership for Advanced Computing National Facilities (APAC). 


\section{References}

Aste, T. 2005. Variations around disordered close packing, J. Phys.: Condens. Matter 17: S2361-S2390.

Aste, T., Saadatfar, M., Sakellariou, A. Senden, T. 2004. Investigating the geometrical structure of disordered sphere packings, Physica A 339: 16-23.

Aste, T., Saadatfar, M. Senden, T. 2005. The geometrical structure of disordered sphere packings, Phys. Rev. E. 71: 061302.

Aste, T. Weaire, D. 2000. The Pursuit of Perfect Packing, Institute of Physics, Bristol.

Ball, R. C. Blumenfeld, R. 2002. Stress field in granular systems: Loop forces and potential formulation, Phys. Rev. Lett. 88: 115505.

Bernal, J. D. Mason, J. 1960. Co-ordination of randomly packed spheres, Nature 188: 910911.

Bideau, D. Hansen, A. (eds) 1993. Disordered and Granular Media, North-Holland, Amsterdam.

Conway, J. Sloane, N. 1997. Low dimensional lattices vii: Coordination sequences, Proc. Royal Soc. London A 453: 2369.

Mason, G. 1968. Radial distribution function from small packings of spheres, Nature 217: 733.

Scott, G. D. 1962. Radial distribution of random close packing of equal spheres, Nature 194: 956-957. 